\title{
Erratum to: 53BP1 depletion causes PARP inhibitor resistance in ATM-deficient breast cancer cells
}

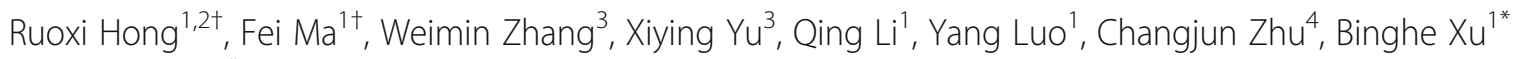
and Wei Jiang ${ }^{3^{*}}$

\section{Erratum}

After the publication of the article [1], the authors realised that the corresponding authors' order need to be changed. Details as follows:

Correct corresponding author order: Binghe $\mathrm{Xu}^{1^{*}}$ and Wei Jiang $3^{3 "}$

Correct correspondence: xubinghe@medmail.com.cn; wjiang6138@cicams.ac.cn

Please note that the above author list and corresponding emails have been updated.

\footnotetext{
Author details

'Department of Medical Oncology, Cancer Institute and Hospital, Peking Union Medical College and Chinese Academy of Medical Sciences, Beijing, China. ${ }^{2}$ Department of Medical Oncology, Sun Yat-sen University Cancer Center, Guangzhou, China. ${ }^{3}$ State Key Laboratory of Molecular Oncology, Cancer Institute and Hospital, Chinese Academy of Medical Sciences and Peking Union Medical College, Beijing, China. ${ }^{4}$ College of Life Science/Tianjin Key Laboratory of Cyto-Genetical and Molecular Regulation, Tianjin Normal University, Tianjin 300387, China.
}

Received: 22 November 2016 Accepted: 22 November 2016

Published online: 30 November 2016

\section{Reference}

1. Hong R, et al. 53BP1 depletion causes PARP inhibitor resistance in ATM-deficient breast cancer cells. BMC Cancer. 2016;16:725. doi:10. 1186/s12885-016-2754-7.

\footnotetext{
*Correspondence: xubinghe@medmail.com.cn; wjiang6138@cicams.ac.cn ${ }^{\dagger}$ Equal contributors

'Department of Medical Oncology, Cancer Institute and Hospital, Peking Union Medical College and Chinese Academy of Medical Sciences, Beijing, China

${ }^{3}$ State Key Laboratory of Molecular Oncology, Cancer Institute and Hospital, Chinese Academy of Medical Sciences and Peking Union Medical College, Beijing, China
} 